\title{
Lyapunov characteristic exponent calculation for finite element discretized models
}

\author{
S. Rugonyi, K.J. Bathe* \\ Massachusetts Institute of Technology, Mechanical Engineering Department, Cambridge, MA 02139, USA
}

\begin{abstract}
In many applications in engineering and science, it is important to know whether the response of a nonlinear system is chaotic. This characterization is possible by the evaluation of the one-dimensional Lyapunov characteristic exponent (LCE). In this paper, a numerical procedure to calculate the LCE of continuous systems discretized using finite element methods is presented.
\end{abstract}

Keywords: Finite element method; Chaotic behavior; Lyapunov characteristic exponent; Nonlinear dynamics; Fluidstructure interaction; Dynamic stability

\section{Introduction}

In the last few decades, the analyses of nonlinear continuous systems have increased due to the availability of faster computers together with the need for more accurate models in engineering and science. Chaotic behavior is found in many nonlinear systems and the prediction and quantification of it can be of major importance.

A chaotic behavior is characterized by an aperiodic long-term response and sensitivity to initial conditions [1]. The system's nearby trajectories in phase space diverge exponentially fast from each other, and as a consequence, the response of a chaotic system is unpredictable. In engineering, a chaotic behavior is usually avoided, but the system response can become regular (non-chaotic) by changing system parameters or the external forcing. In either case, it is necessary to know the nature of the response in order to change it.

The Lyapunov characteristic exponent (LCE) is associated with the asymptotic dynamic stability of the system: it is a measure of the exponential divergence of trajectories in phase space. The exponent provides a means of ascertaining whether the behavior of a system is chaotic.

An approach for the numerical calculation of the LCE of discrete dynamical systems and maps was given by

\footnotetext{
* Corresponding author. Tel.: +1 (617) 253-6645; Fax: +1 (617) 253-2275; E-mail: kjb@mit.edu
}

Benettin et al. [2,3]. We used this approach as a basis for the development of a numerical technique to calculate the LCE of continuous media discretized using finite element methods, as presented in this paper.

\section{Lyapunov characteristic exponent}

Consider the following system of nonlinear non-dimensional equations

$\dot{\mathbf{x}}=\mathbf{f}(\mathbf{x})$

with initial conditions

$\mathbf{x}\left(t_{0}\right)=\mathbf{x}_{0}$

We are interested in the behavior of small perturbations to the reference trajectory $\mathbf{x}_{r}(t)$, which is the solution of Eqs. (1) and (2). The equation describing the time evolution of perturbations, $\mathbf{y}$, is obtained by linearizing Eq. (1) about $\mathbf{x}_{r}(t)$,

$\dot{\mathbf{y}}=\left.\frac{\partial \mathbf{f}}{\partial \mathbf{x}}\right|_{\mathbf{x}_{r}(t)} \mathbf{y}$

where $\partial \mathbf{f} / \partial \mathbf{x}$ is the Jacobian matrix of the function $\mathbf{f}(\mathbf{x})$, which is assumed to be sufficiently smooth.

The LCE of a response is defined as

$\chi=\lim _{t \rightarrow \infty} \frac{1}{t} \ln \frac{\|\mathbf{y}(t)\|}{\left\|\mathbf{y}_{0}\right\|}$ 
where $\mathbf{y}_{0}$ is an arbitrary initial perturbation, and $\chi$ is positive when the behavior is chaotic. For all $\mathbf{x}_{0}$ lying in the basin of attraction of an attractor (such as a fixed point, limit cycle or chaotic attractor), the LCE does not depend on the reference trajectory, $\mathbf{x}_{r}$, nor on the choice of the initial perturbation, $\mathbf{y}_{0}$, see for example Benettin et al. [3].

The perturbation vector can be expressed by

$\mathbf{y}(t)=\boldsymbol{\Phi}\left(t, t_{0}\right) \mathbf{y}_{0}$

where $\boldsymbol{\Phi}\left(t, t_{0}\right)$ is a mapping matrix with the property that for $t_{2}>t_{1}>t_{0}$

$\mathbf{y}\left(t_{2}\right)=\boldsymbol{\Phi}\left(t_{2}, t_{1}\right) \boldsymbol{\Phi}\left(t_{1}, t_{0}\right) \mathbf{y}_{0}$

Approximations to the matrices $\boldsymbol{\Phi}\left(t_{n}, t_{n-1}\right)$ can be formally obtained by discretizing Eq. (3) in time and inverting the obtained left-hand side coefficient matrix. Notice that in a numerical calculation the perturbation vector, $\mathbf{y}\left(t_{n}\right)$, must be normalized after a given number of time steps since otherwise the norm of the vector might grow (exponentially fast) and overflow might occur.

The following procedure can be followed to calculate the LCE:

1. Start with an initial perturbation such that $\tilde{\mathbf{y}}_{0}=\mathbf{y}_{0} /\left\|\mathbf{y}_{0}\right\|$

2. For each time step $i$ calculate

$$
\begin{aligned}
\mathbf{y}_{i}^{*} & =\boldsymbol{\Phi}\left(t_{i}, t_{i-1}\right) \tilde{\mathbf{y}}_{i-1} \\
d_{i} & =\left\|\mathbf{y}_{i}^{*}\right\| \\
\tilde{\mathbf{y}}_{i} & =\frac{\mathbf{y}_{i}^{*}}{d_{i}}
\end{aligned}
$$

Note that $\left\|\mathbf{y}\left(t_{i}\right)\right\|=d_{i} \cdot d_{i-1} \cdot \ldots \cdot d_{1}$

3. Calculate successive approximations of the LCE as a function of time

$$
k_{n}=\frac{1}{t_{n}} \sum_{i=1}^{n} \ln d_{i}
$$

where $t_{n}=t_{0}+n \Delta t$, and $\Delta t$ is the time step employed, and $t_{0}$ is the initial time.

Note that, using the numerical procedure described above,

$$
\chi=\lim _{\substack{\Delta t \rightarrow 0 \\ n \rightarrow \infty}} k_{n}
$$

\section{Calculation of LCEs of discretized finite element models}

Let us now focus on the calculation of the LCE of a continuous system discretized using finite element methods.

The linearized finite element discrete equations of a nonlinear structure can be written as [4]

$$
\mathbf{M u}+\mathbf{C} \dot{\mathbf{u}}+\mathbf{K u}=\mathbf{R}-\hat{\mathbf{F}}
$$

where $\mathbf{u}$ is the vector of incremental nodal point displacements (and can include other degrees of freedom such as pressures or rotations depending on the finite element formulation employed), $\mathbf{M}, \mathbf{C}$ and $\mathbf{K}$ are the mass, dissipation and stiffness matrices, respectively, $\mathbf{R}$ is the load vector and $\hat{\mathbf{F}}$ contains known terms from the linearization process. The corresponding equations for the evolution of perturbations are

$$
\mathbf{M} \ddot{\tilde{\mathbf{u}}}+\mathbf{C} \dot{\tilde{\mathbf{u}}}+\mathbf{K} \tilde{\mathbf{u}}=\mathbf{0}
$$

where $\tilde{\mathbf{u}}$ is the vector of perturbations. At each time step, Eqs. (10) and (11) can be solved to obtain the reference trajectory and the evolution of the perturbation.

Steps 1, 2 and 3 described in the last section could be followed to calculate the successive values of $k_{n}$. However, since $\mathbf{y}^{T}=(\tilde{\mathbf{u}} \dot{\tilde{\mathbf{u}}})$, it is necessary to non-dimensionalize the perturbed displacements and velocities to calculate the norm of the perturbation vector $\mathbf{y}$, that is

$d_{i}=\sqrt{\sum_{j=1}^{N} \frac{\tilde{u}_{j}^{2}}{L^{2}}+\sum_{j=1}^{N} \frac{\dot{\tilde{u}}_{j}^{2}}{\omega^{2} L^{2}}}$

where $N$ is the total number of structural degrees of freedom, $\tilde{u}_{j}$ and $\dot{\tilde{u}}_{j}$ are the components of $\tilde{\mathbf{u}}$ and $\dot{\tilde{\mathbf{u}}}$ at time $t_{i}$, respectively, and $L$ and $\omega$ represent a characteristic length and frequency of the problem. It is evident that the characteristic length plays only the role of a scaling constant; in contrast, the correct choice of the value of $\omega$ is essential to the determination of the appropriate norm. Considering a system that does not have a chaotic response (such as a linear system), it is observed that when $\omega$ is chosen so that $\omega<\omega_{\max }$, with $\omega_{\max }$ the maximum frequency of the finite element model, the norm of the perturbation, $d_{i}$, usually grows over a long period of time leading to the wrong conclusion that the system is chaotic. A detailed analysis of this situation and the solution proposed will be published in a future paper [5]. To avoid a spurious growth of the perturbation vector norm, the velocity has to be non-dimensionalized using a frequency that is greater than or equal to the maximum frequency of the finite element model, i.e. $\omega \geq \omega_{\max }$ in Eq. (12). However, $\omega_{\max }$ can be a very large number, in which case the contribution from the perturbation velocity vector is negligible in Eq. (12), leading to

$d_{i}=\sqrt{\sum_{j=1}^{N} \frac{\tilde{u}_{j}^{2}}{L^{2}}}$

Comparisons of the results obtained with Eq. (13) and Eq. (12) in the analysis of low order systems reveals that using Eq. (13) the local maximum values of the calculated $k_{n}$ values match the values obtained using Eq. (12). As a consequence, if Eq. (13) is used, the LCE is calculated as

$$
\chi=\limsup _{\substack{\Delta t \rightarrow 0 \\ n \rightarrow \infty}} k_{n}
$$

The ideas described above for structural problems discretized using finite element methods can be used in the 
analysis of fluid-structure interaction systems in which only the behavior of the solid part is of interest. In such a case, all the equations (from the fluid and structure) are considered in the calculation of the perturbation, but the norm $d_{i}$ is calculated using only the perturbed displacements of the structure [6].

\section{Numerical examples}

\subsection{Buckled beam}

Fig. 1 shows the system analyzed, which consists of a buckled beam with applied periodic forcing. It is well known that for certain frequencies and amplitude of excitation, the beam behavior becomes chaotic [7]. The beam

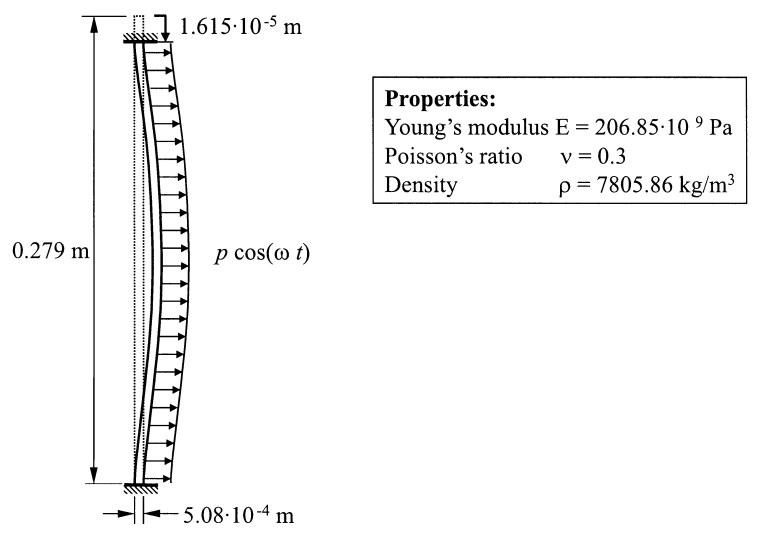

Fig. 1. Buckled beam problem considered.

a)

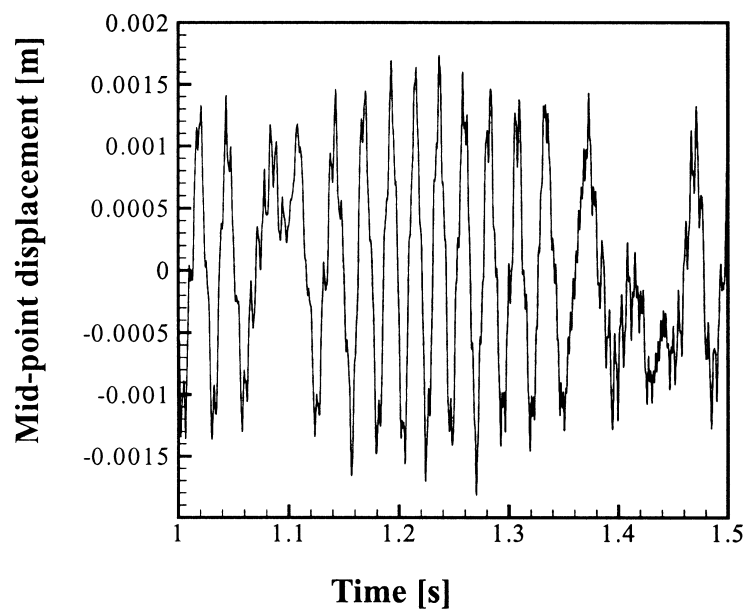

was analyzed using an excitation frequency of $90 \mathrm{~Hz}$ and amplitude of excitation of $p=45 \mathrm{~Pa}$, and discretized using $80 \times 19$-node plane stress elements. Experimental results for the beam are presented in [8]. The chaotic response together with the calculated values of the approximations to the LCE, $k_{n}$, are shown in Fig. 2.

\subsection{Collapsible channel}

The model of the channel with fluid flow analyzed is presented in Fig. 3. A part of the upper channel wall was replaced by a membrane that can displace in the horizontal and vertical directions (the collapsible segment), followed by another part that can only displace horizontally. Fig. 3 also shows the membrane behavior, which exhibits a limit cycle response. The system LCE, which goes to zero since the membrane behavior is not chaotic, is shown in Fig. 4. This example serves as a test for the LCE calculation.

\section{Conclusions}

In the analysis of nonlinear dynamic systems, different types of responses can occur. In particular, to ascertain whether the response is chaotic, the LCE can be evaluated. Since a chaotic behavior is sensitive to initial and model conditions, different numerical discretizations may lead to different response solutions, but the value of the LCE indicates the chaotic nature of the behavior.

This paper presents a numerical procedure for the calculation of the LCE of continuous systems discretized using finite element methods. In the proposed algorithm, all discretized degrees of freedom (and therefore all dis-

b)

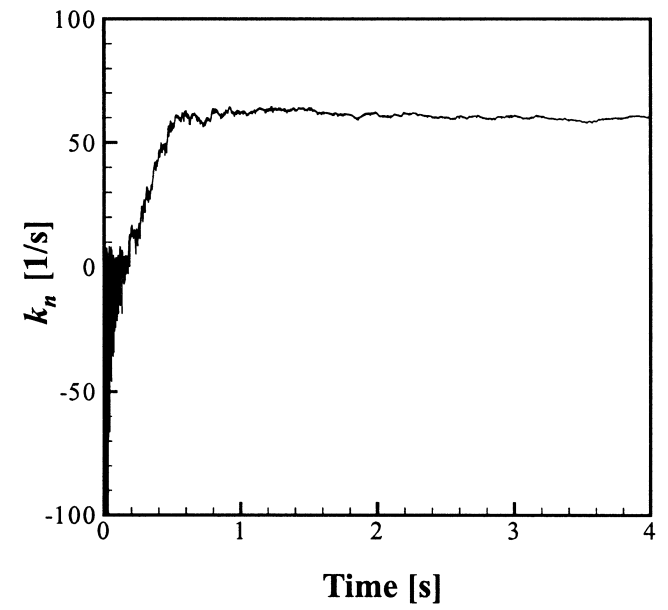

Fig. 2. (a) Buckled beam dynamic response. (b) Calculated Lyapunov characteristic exponent. 

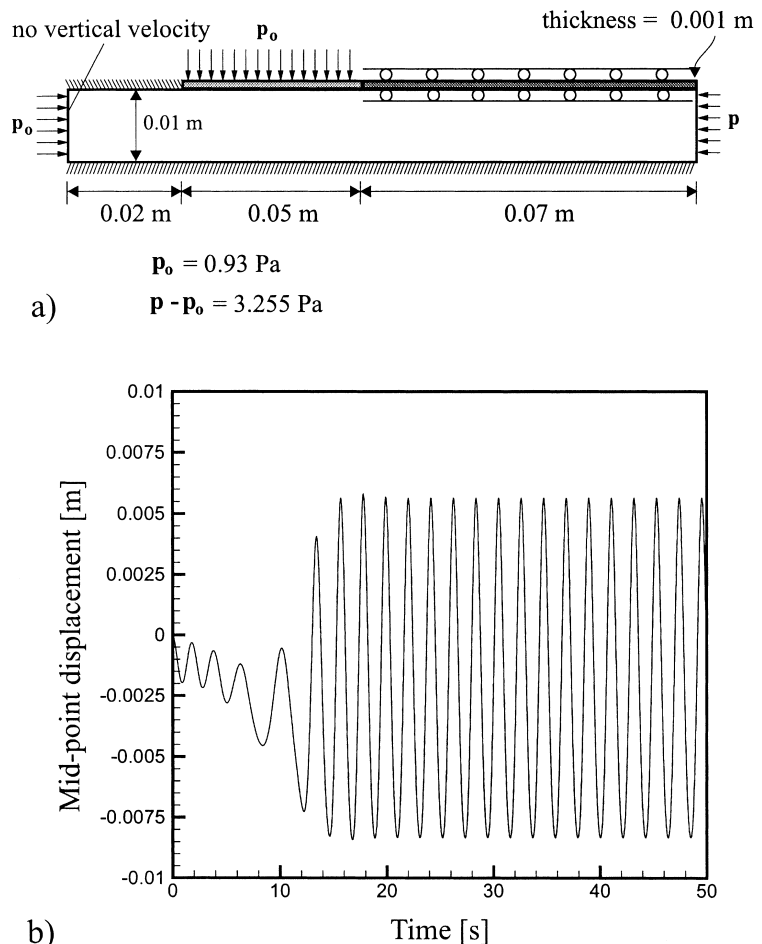

Fig. 3. (a) Collapsible channel problem considered. NavierStokes fluid with structure. (b) Obtained mid-point membrane displacement.

crete modes) are perturbed, and the growth/decay of the perturbation in time is calculated by means of linearized evolution equations.

The computational cost of the algorithm is approximately equivalent to an additional iteration step in a Newton-Raphson iteration procedure, and therefore calculating the LCE typically added about $25 \%$ of the total computation time for the examples presented here.

\section{Acknowledgements}

The authors are very thankful for the Rocca Fellowship supporting this work.

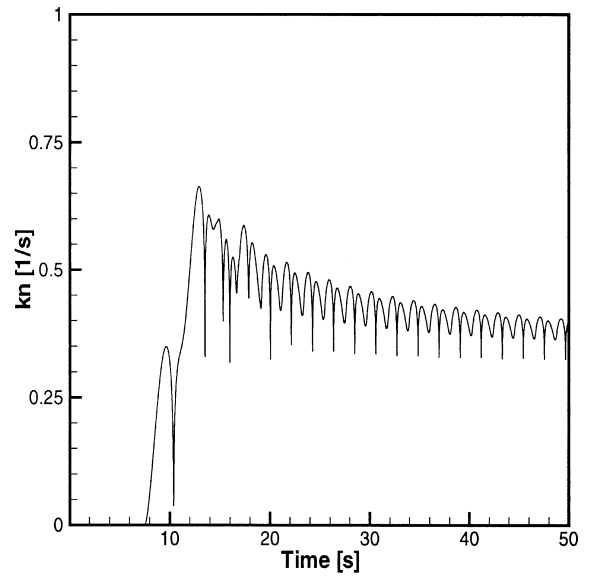

Fig. 4. Calculated Lyapunov characteristic exponent for the collapsible channel problem. Since the system response is not chaotic, the LCE converges to zero.

\section{References}

[1] Strogatz SH. Nonlinear Dynamics and Chaos, Addison-Wesley, 1994.

[2] Benettin G, Galgani L, Strelcyn JM. Kolmogorov entropy and numerical experiments. Phys Rev A 1976;14:23382345.

[3] Benettin G, Galgani L, Giorgilli A, Strelcyn JM. Lyapunov characteristic exponents for smooth dynamical systems and for hamiltonian systems; a method for computing all of them. Part 1: Theory. Part 2: Numerical applications. Meccanica 1980;15:9-30.

[4] Bathe KJ. Finite Element Procedures, Prentice-Hall, New York, 1996.

[5] Rugonyi S, Bathe KJ. An evaluation of the Lyapunov characteristic exponent of chaotic continuous systems, in preparation.

[6] Rugonyi S, Bathe KJ. On finite element analysis of fluid flows fully coupled with structural interactions. Comput Model Eng Sci, in press.

[7] Moon FC. Chaotic Vibrations, John Wiley and Sons, 1987.

[8] Kreider W, Nayfeh AH. Experimental investigation of singlemode responses in a fixed-fixed buckled beam. Nonlinear Dynamics 1998;15:155-177. 